\title{
Los nuevos retos de la Educación General universitaria en el siglo XXI
}

\section{The new challenges of General University Education in the 20th Century}

\author{
Waldemiro Vélez Cardonal \\ Universidad Nacional, Sede Central \\ Heredia, Costa Rica \\ waldemirov@hotmail.com
}

Resumen: Se presentan y discuten los principales retos de la Educación General universitaria en el siglo XXI. En primer lugar, superar la extraordinaria influencia que hasta ahora ha tenido el canon occidental, con su metafísica excluyente y a-histórica y acoger el pensamiento decolonial a partir de una apertura epistemológica a saberes que hasta ahora habían sido excluidos de los currículos universitarios. En segundo lugar, integrar plenamente el pensamiento complejo y la perspectiva transdisciplinaria en sus ofrecimientos.

Palabras clave: Estudios Generales, pensamiento decolonial, transdisciplinariedad, complejidad, canon occidental.

Abstract: The main challenges of General university Education in the 21 st century are presented and discussed. First of all, to overcome the strong influence that the western model has had so far, with its excluding and ahistorical metaphysics and to adopt the decolonial concept based on an epistemological opening to knowledge that had until now been excluded from the university curricula. Secondly, to fully integrate complex thinking and the transdisciplinary perspective in its offerings.

1Catedrático en el Departamento de Ciencias Sociales de la Facultad de Estudios Generales en el Recinto de Río Piedras de la UPR y Coordinador de la Red Internacional de Estudios Generales (RIDEG). 
Keywords: General Studies, decolonial concept, transdisciplinarity, complexity, occidental model.

\section{Introducción}

$O$ i bien la segunda mitad del siglo XX estuvo plena de retos para la Educación General, los comienzos del XXI han mantenido los retos del pasado y sumado otros nuevos más trascendentales. Para que la Educación General salga fortalecida de estos, tanto en términos de su reconocimiento y vitalidad intelectual, como en su espacio en la estructura curricular y organizacional de las universidades, se requieren abordajes novedosos en su renovación teórica y filosófica, así como en sus fundamentos y prácticas pedagógicas para que sean más relevantes y estimulantes, sobre todo para los estudiantes.

En ese contexto, se hace imperativo reconocer e incorporar la diversidad -en todas sus manifestaciones- para integrar los planteamientos epistemológicos que proponen un conocimiento situado, del margen o frontera, diferente del que hasta entonces ha sido hegemónico. Esto requiere que vayamos dejando atrás la noción de que el conocimiento es el mismo en todo tiempo y espacio, idea que por muchos años sirvió de fundamento a los defensores del Canon Occidental como fundamento de la Educación General. También implica que incorporemos las teorías de la complejidad y la concepción transdisciplinaria del mundo, en la nueva Educación General que deseamos desarrollar. Ese es, a mi juicio, un reto que debemos abordar colaborativamente incorporando experiencias y saberes que hasta ahora habían permanecido marginados e invisibilizados, por no ser "científicos" o "académicos". Para ello, necesitamos desarrollar las "tecnologías de humildad", como ha planteado Sheila Jasanoff (2003 y 2007) y, de alguna manera, reinventarnos como docentes. Lo que de por sí representa un reto de grandes proporciones.

\section{Los principales retos de la Educación General}

Habiendo concluido la primera década del siglo XXI, debemos avanzar significativamente en la evaluación de los modelos de Educación General más utilizados por las universidades. El debate entre los modelos prescriptivos ${ }^{2}$-que algunos identifican con la defensa del Canon Occidental-, por una parte, y los

2 En este modelo las autoridades universitarias deciden e imponen cuáles son los saberes que todos los estudiantes deben poseer y qué cursos universitarios tienen que tomar para obtenerlo. 
modelos distributivos ${ }^{3}$ que no han logrado solucionar el problema de la coherencia y la integración, por otra, condujo al estancamiento de la Educación General. Muchas universidades intentan resolver este problema mediante estructuras curriculares híbridas en las que se inserta un poco de prescripción (de seis a ocho cursos regularmente), al interior de modelos mayormente distributivos (Lattuca \& Stark, 2009, p.32). Esta modalidad tampoco ha tenido los resultados esperados (Gaff, 1983; Ratcliff, 1997; Johnson \& Ratcliff, 2004 y Boning, 2007).

La excesiva identificación de la Educación General con una de sus modalidades (la prescripción fundamentada en el Canon Occidental) ha conducido a muchas personas, incluyendo a los responsables de las políticas públicas sobre educación superior, a pensar que la Educación General está pasada de moda y que ha perdido toda su pertinencia, por lo que no se justifica continuar dedicándole unos recursos cada vez más escasos y competitivos. Uno de los problemas que ha enfrentado la Educación General es que, a través de los años, ha tenido malos defensores ${ }^{4}$. Éstos, en lugar de apuntalar la vigencia y mayor pertinencia de esta, han conducido a todo lo contrario.

Por un lado, están los que defienden la Educación General como la única capaz de regresar a un pasado idílico en el que, por medio de los clásicos o aquellos que tienen vigencia en cualquier época y lugar, los estudiantes universitarios recibían la mejor educación posible. Por otro lado, se encuentran quienes afirman que la Educación General está en todo, por lo que no hay que hacer nada deliberado ni coherente en el programa de estudios para fortalecerla. Según ellos, casi cualquier curso se podría denominar de Educación General.

A mi juicio, lo que le otorga plena actualidad a la Educación General es que dicho componente del currículo universitario es el más afín con los saberes de punta, que están en los bordes, y más allá de las fronteras disciplinarias; los saberes que expanden nuestra noción de la realidad, del conocimiento y de la vida misma. Es desde la periferia, no desde el centro canónico, donde se puede incorporar plenamente el pensamiento complejo y la concepción transdisciplinaria del mundo (Morín, 2002, 2003 y 2004; Nicolescu, 1998 y 2002). Por tanto, la defensa de la Educación General debe alejarse del Canon Occidental y de las perspectivas que la ubican como algo que se logra sin un esfuerzo deliberado.

3 En este caso a los estudiantes se les permite escoger los cursos que pre eran, entre un conjunto (menú) de alternativas, al interior de cada una de las principales áreas del saber (ciencias sociales, ciencias naturales y humanidades, incluyendo la literatura y los idiomas).

4 Ver, entre muchos otros, a Bloom (1987), Bennett (1984), y Cheney (1989). 
Según Rhoads (1995, p. 260), la naturaleza jerárquica del Canon silencia la diversidad cultural. Lo que se selecciona como parte del Canon involucra un juicio de valor acerca de la calidad o estética de trabajos, ideas, modos de conocer y formas de conocimiento específicos. Por esta razón, el Canon debe ser entendido como una forma de ideología que ayuda a suprimir todo conocimiento que se ubica fuera del "mainstream."Hacia una Educación General postcolonial, no canónica y occidentalista

La academia "occidental" generalmente permanece ensimismada y casi nunca incorpora conocimientos provenientes de otras tradiciones filosóficas y culturales, aún cuando el potencial de aprendizaje epistemológico puede ser extraordinario. Sería muy interesante considerar el indicador de Felicidad Nacional Bruta que se utiliza en Bhutan, el que está basado en los valores budistas y tiene importantes implicaciones para la relación entre la organización social y la protección del medio (Woiwode, 2013, p. 398). Para esto haría falta acoger el discurso decolonial ${ }^{5}$, en el cual se valoran otros estilos de vida y de pensamiento que marcan continuamente la diferencia con el proceso de occidentalización (Mignolo, 1995, p. 32). Según Walter Mignolo (1995, p. 37 y 39):

...las teorías postcoloniales pueden convertirse en un instrumento útil para la reorganización del saber, la política cultural y la programación curricular, medios particulares de llevar adelante la descolonización intelectual; o, si se quiere, de tomar en serio el hecho de que los "orígenes" no están en Grecia sino en cualquier lugar. Esto es, de tomar en serio la política de la ubicación geocultural como la ética y la política de la enunciación...En todo caso, el lugar de la crítica y teoría postcoloniales sería el de la permanente construcción de lugares de enunciación en los marcosdiscursivos construidos por los sucesivos momentos del proceso de occidentalización: desde la expansión mercantilista hasta la globalización pasando por la revolución industrial y la expansión capitalista.

En el siglo XX se hicieron descubrimientos científicos importantes que ayudaron a develar la complejidad y la interconectividad, como aspectos definitorios del universo y de las relaciones sociales. Sobre todo, la noción de interconexión ha sido parte esencial de la sabiduría en las tradiciones culturales ancestrales. Su revaloración puede ser de mucha ayuda para encontrar la solución a problemas tan urgentes como el cambio climático, entre muchos otros. El pasado siglo fue testigo de la convergencia entre la ciencia y las sabidurías ancestrales, por medio

5 Aquí entendemos la colonialidad como un discurso y una práctica que simultáneamente establece la inferioridad natural de los sujetos y la colonización de la naturaleza, lo que define a ciertos sujetos como dispensables y a la naturaleza como pura materia prima para la producción de bienes para el mercado internacional (Maldonado-Torres, N., 2007, p. 135). 
del entendimiento, el cual dio lugar a concepciones del mundo complejas, en lugar de las que se basaban en un sistema mecánico. Esta visión del mundo ahora se fundamenta, entre otros aspectos, en la no-linealidad en las interrelaciones causales complejas (bucles retroactivos, co-evolución, emergencia), en la que se ha borrado la separación en la relación sujeto-objeto, y donde se reconoce a los sistemas como autopoiéticos. Uno de los avances más significativos de la física cuántica fue, probablemente, el re-descubrimiento de la no-separabilidad como un tipo de causalidad, algo que no es nuevo para el pensamiento hindi o budista, entre otros (Woiwode, 213, pp. 387-388).

Según Henagulph (2000, p. 3), la inseparabilidad de las dualidades era parte del entendimiento del mundo que se desarrollaba en varias culturas, incluyendo la Celta, la de los Nativos-americanos y la Taoísta. Estas reconocían el valor de la paradoja para el desarrollo del pensamiento y entendían el siguiente planteamiento de Thomas Mann: "Una gran verdad es una verdad cuyo opuesto también es una gran verdad." En la tradición Budista Zen se utilizan planteamientos o historias paradógicas (Koan) como mecanismo pedagógico para estimular, por medio de la meditación y el pensamiento intuitivo que se desprende de ella, la comprensión de las aparentes contradicciones (Wickson, Carew y Russell, 2006, pp. 10541055; Max-Neef, 2005, p. 12).

Para Woiwode (2013), unir la psicología occidental con la filosofía y la práctica de las sabidurías ancestrales podría contribuir a la solución de muchas crisis contemporáneas, e incorporar técnicas de crecimiento espiritual que van más allá de lo considerado en occidente, pudiendo penetrar niveles más profundos de la mente y del alma, con el potencial de superar las fronteras cognitivas que la ciencia occidental (que por mucho tiempo las ha considerado esotéricas e irracionales) no ha logrado superar (p. 390).

La experiencia Satori (la experiencia intuitiva interior que conduce a la ilustración del ser), utilizada en el Budismo y el Taoísmo, podría ser de mucha utilidad para activar niveles de percepción con los que hasta ahora no hemos podido estar en contacto. En Mesoamérica esto se ha trabajado por siglos en los rituales Shamánicos, en los cuales se inducen estados alterados de la consciencia, de los que tenemos mucho que aprender (Max-Neef, 2005, p. 13). En síntesis, como afirman Plaz y Vessuri (2007, p. 11):

6 Citado en Wickson, Carey y Russell (2006, p.1054). 
...será necesario aprender a integrar culturas diferentes en el modo de relacionarse, producir y diseminar el saber construido. Esto no parece haberlo comprendido muchos de los actores de uno y otro ámbito social ya que las tensiones sociopolíticas son uno de los principales obstáculos presentes que afectan el diálogo intercultural. El desafío presente está en aventurarnos a indagar, en estos espacios sociales dinámicos de aprendizaje intercultural y transdisciplinario que tienden a promoverse en el sector académico público y privado, cómo se construyen los nuevos modelos de producción del saber, del hacer, del convivir.

Los recursos institucionales deben utilizarse para desarrollar espacios de aprendizaje transdisciplinarios e interculturales. De esa forma se podrá contribuir a una sociedad de saberes compartidos por medio del diálogo y la deliberación con actores y culturas que han permanecido excluidos, por muchos años, para recuperar los valores de paz y preservación ambiental que, de muchas maneras, se han debilitado en la tradición intelectual occidental (Op.cit. p.12).

Como brillantemente ha planteado Catherine Walsh (2007, p. 33):

Pensar en un giro o, mejor dicho, un vuelco decolonial en torno al conocimiento y a la educación requiere tomar con seriedad tanto las contribuciones como las implicaciones de historias locales y de epistemologías negadas, marginalizadas y subalterizadas. Requiere también una atención a la formación de conexiones dialógicas entre ambas. Pero tal vez más importante aún, requiere una atención política y ética a nuestras propias prácticas y lugares de enunciación con relación a estas historias y epistemologías...

Soy de los que piensa que la universidad es el espacio social apto para privilegiar aquellos procesos educativos que nos permitirán desarrollar aprendizajes decoloniales, transdisciplinarios e interculturales, al propiciar la construcción de nuevos modelos de producción del saber, del conocimiento, del hacer y del ser, a partir de nuevas maneras de convivir. Mi principal planteamiento aquí es que la Educación General es el componente de los currículos universitarios que mejor puede incorporar las sabidurías ancestrales y ayudar a producir conocimientos socialmente robustos. Esto porque su razón de ser es propiciar la re-vinculación holística de los saberes, y reconocer la complejidad de nuestra existencia y la unidad intrínseca de todos los elementos que la configuran y posibilitan (biológicos, químicos, socioculturales, medioambientales, físicos, emocionales, espirituales, entre otros.). Por eso, debe incorporar la transdisciplinariedad como la estrategia más adecuada para producir aprendizajes, conocimientos, culturas, acciones y maneras de vivir y de ser que produzcan el mayor bienestar individual $\mathrm{y}$, sobre todo, colectivo. 
La Educación General, al igual que la transdisciplinariedad, se caracteriza por propiciar la conexión e integración de saberes provenientes de los diversos campos del conocimiento (ciencias sociales, naturales y humanidades), y de incorporar integralmente las experiencias y vivencias cotidianas de los estudiantes en los procesos de aprendizaje (Dewey). De esa manera, se integran los saberes científicos con la intuición, la sensibilidad y toda forma de producir conocimientos y sabiduría que poseemos como herencia, particularmente, aquellos que han sido olvidados o escondidos (los saberes indoamericanos, orientales, africanos, entre otros), los que, como hemos visto, son extremadamente importantes para superar barreras cognitivas y producir nuevos conocimientos que nos ayuden a solucionar problemas apremiantes de nuestro tiempo.

Al ubicarnos, docentes y estudiantes, como sujetos en permanente transformación, podemos aceptar y valorar las preguntas abiertas y sin respuesta; los espacios no saturados, los órdenes siempre incompletos; a la vez, que se disfruta la relación compleja entre la certeza y la incertidumbre que habitamos y nos habita, al aceptar la presencia de vacíos, pues sabemos que a través de ello con frecuencia viene el cambio (Najmanovich, 2006, p. 13).

Además, la Educación General ha sido y es el componente de los currículos universitarios que ha tenido la principal responsabilidad de promover la conciencia ciudadana. Pero, ahora me refiero no a una ciudadanía excluyente y con pretensiones homogeneizadoras, sino a una ciudadanía intercultural y compleja (Tubino, 2003). Es decir, a unas nociones y prácticas ciudadanas sensibles a nuestras diferencias étnicas, raciales, idiomáticas, de género, religión, preferencias sexuales, posturas políticas y quehacer cultural, entre otros. En ese contexto, la Educación General promueve el desarrollo de la tolerancia, la empatía, la solidaridad y la afectividad; a la vez, ayuda a desarrollar competencias cognitivas e intelectuales de alto nivel. En todo momento debemos tener presente que la posibilidad del ejercicio pleno de la ciudadanía tiene varios prerrequisitos multidimensionales, que están profundamente interconectados entre sí. De ahí la importancia de la Educación General transdisciplinaria, tanto para el entendimiento de estos nexos, como para propiciar prácticas cotidianas que aumenten las posibilidades del ejercicio de una ciudadanía compleja y plural, que conduzca a la ampliación de los espacios democráticos.

El componente curricular en el que cohabitan, de manera integrada, la literatura, el arte, la ciencia, la historia, la filosofía, y el compromiso con el desarrollo de una ciudadanía intercultural y democrática, como vimos, es la Educación General. Es a 
partir de esta como mejor podremos emprender el mayor reto de nuestro tiempo, una reforma del pensamiento. Esta es la que, en palabras de Morin (2010):

...permitiría frenar la regresión democrática que suscita, en todos los campos de la política, la expansión de la autoridad de los expertos, especialistas de todos los órdenes. Esa expansión ha estrechado progresivamente la competencia de los ciudadanos, condenados a la aceptación ignorante de las decisiones tomadas por quienes se presupone que saben, pero que de hecho practican una inteligencia ciega, por parcial y abstracta, que fragmenta la globalidad y pierde de vista el contexto de los problemas. El desarrollo de una democracia cognitiva sólo (sic) es posible mediante una reorganización del saber, la cual exige una reforma del pensamiento que permitiría no solamente separar para conocer, sino también religar lo que se separa. Se trata de una reforma más profunda y amplia que la de una democratización de la enseñanza universitaria y una generalización del estado de estudiante. Se trata de una reforma, no ya programática, sino paradigmática, que concierne a nuestra aptitud para organizar el conocimiento (pp.12-13)

Para Tamariz y Espinoza (2007, p. 22), la cosmovisión transdisciplinaria es ella misma integral, pues incorpora al cosmos, al hombre espiritual y al hombre situado en el mundo. Es el resultado de la visión sintética de todas las disciplinas, tanto las que tradicionalmente se han denominado como ciencias naturales, sociales y humanísticas; pero también es producto de otros modos de conocer, los que dejaron de ser predominantes, pero que están vivos aún y aportan a entender dimensiones humanas, que de otra manera no estarían accesibles (la espiritualidad, nuestro ser interior, todo aquello que se ubica en lo sagrado, porque escapa a la razón).

Nuestro gran reto en la actualidad es lograr una reforma de la universidad que nos ayude a reconstruir la unidad y la pertinencia del conocimiento, para restaurar la búsqueda de sabiduría como el eje central de la misión de la universidad. La sabiduría es eminentemente transdisciplinaria, no puede estar contenida en ninguna disciplina académica especializada o combinación de estas. Tampoco se puede obtener por medio del trabajo académico -como lo conocemos hoysolamente (Weislogel, 2011, p.223). 


\section{Conclusiones}

Hoy, somos cada vez más conscientes de la naturaleza contingente, parcial, preliminar y contextual del conocimiento, gracias a las importantes aportaciones, tanto de la ciencia como de la filosofía del siglo XX. Pensamos en Einstein, Bohr, Heisenberg, Godel, Tarski, Foucault, Derrida, Rorty, Tagore (India), Mariátegui (Perú), entre muchos otros. Dicha aportación ha sido incorporada y elaborada por las teorías de la complejidad, las que han producido una devastadora crítica de la metafísica moderna y de los binarismos característicos de la decadente episteme occidental, en los que se fundamentaba el "Canon".

La Educación General, la que es ontológicamente afín con el pensamiento complejo y con la concepción transdisciplinaria del conocimiento y del mundo, es el componente del currículo universitario mejor equipado para asimilar la ruptura epistemológica que se llevó a cabo sobre todo en la segunda mitad del siglo $\mathrm{XX}$. Ahí se encuentran, a mi juicio, los fundamentos teóricos y epistemológicos de su renovación. Al emprender su renovación teórica y práctica a partir de la transformación de los saberes, la cual se llevó a cabo después de la segunda mitad del siglo XX, podremos demostrar la vigencia y prioridad que debe tener en los estudios universitarios del siglo XXI. Necesitamos convencer a todos los constituyentes de que la Educación General es la mejor inversión de los escasos fondos universitarios, así como la mejor manera de propiciar el aprendizaje en la universidad contemporánea. Estos son nuestros principales retos.

\section{Referencias}

Bennett, W.J. (1984). To Reclaim a Legacy: A Report on the Humanities in Higher Education. Washington: National Endowment for the Humanities. http://files.eric. ed.gov/fulltext/ED247880.pdf

Bloom, A. (1987). The Closing of the American Mind. New York. Simon \& Schuster

Boning, K. (2007). Coherence in General Education: A Historical Look. The Journal of General Education, 56(1), 1-16. http://dx.doi.org/10.1353/jge.2007.0008

Cheney, L. (1989). 50 Hours: A Core Curriculum for College Students. Washington DC: National Endowment for the Humanities.

Gaff, J. G. (1983). General Education Today: A Critical Analysis of Controversies, Practices, and Reforms. San Francisco: Jossey-Bass Publishers.Change: The Magazine of Higher Learning Volume 15, Issue 6, 1983 
Henagulph, S. (2000). Three Pillars of Transdisciplinarity. Montréal. Recuperado de: http://www.goodshare.org/pillars.htm.

Jasanoff, S. (2007, November 1). Technologies of humility. Nature, 450, 33. http://dx.doi. org/10.1038/450033a

Johnson, D. K. \& Ratcliff, J. L. (2004, Spring). Creating Coherence: The Unfinished Agenda. New Directions for Higher Education, (125), 85-95. http://dx.doi. org/10.1002/he.141

Lattuca, L. R. \& Stark, J.S. (2009). Shaping the College Curriculum. Academic Plans in Context (2a ed.). San Francisco: Jossey-Bass Publishers.

Max-Neef, M.A. (2005, March). Foundations of Transdisciplinarity. Ecological Economics, 53, 5-16. http://dx.doi.org/10.1016/j.ecolecon.2005.01.014

Maldonado-Torres, N. (2007). Sobre la colonialidad del ser: contribuciones al desarrollo de un concepto. En: Castro-Gómez, S. y Grosfoguel, R. (Eds.). El giro decolonial. Reflexiones para una diversidad epistémica más allá del capitalismo global, 127167. Bogotá, Colombia: Siglo del Hombre Editores.

Mignolo, W.D. (1995). Occidentalización, imperialismo, globalización: herencias coloniales y teorías postcoloniales. Revista Iberoamericana, 61, (170-171), 27-40. http://dx.doi.org/10.5195/reviberoamer.1995.6392

Morin, E. (2010). Prólogo. A propósito de los saberes globales y fundamentales de la ense-anza universitaria. En Morin, E. (Dir.), Malinowski, N. y Delgado, C. En la ruta de las reformas fundamentales, 9-14. Hermosillo, México: Multiversidad Mundo Real Edgar Morin.

Morin, E. (2004). La epistemología de la complejidad, en Gazeta de Antropología, (20), Texto 20-02.

Morin, E. (2003). Introducción al pensamiento complejo. Barcelona: Gedisa Editorial.

Morin, E. (2002). Los siete saberes necesarios para la educación del futuro. Buenos Aires: Nueva Visión.

Najmanovich, D. (2006). Metamorfosis de fin de siglo: crisis, cambio y complejidad. Conociendo el conocimiento del conocimiento, 13p. Recuperado de: www. denisnajmanovich.com 
Nicolescu, B. (2002). Manifesto of Transdisciplinarity. Albany, NY: State University of New York Press.

Nicolescu, B. (1998). The Transdisciplinary Evolution of the University: Condition for Sustainable Development. Bulletin Interactif du Centre Internacional de Recherches et Ẻtudes Transdiciplinaires, (12), 7p.

Pascal, Blaise (1940). Pensamientos. Madrid: Espasa Calpe.

Piaget, J. (1972). The Epistemology of Interdisciplinary Relationships. In Interdisciplinarity: Problems of Teaching and Research in Universities. Paris: Center for Educational Innovation, 127-139.

Plaz, I. y Vessuri, H. (2007). Espacios para el aprendizaje intercultural y transdisciplinario en una sociedad en transformación. Polis, 16. Recuperado de: http://polis.revues.org/4651

Ratcliff, J. L. (1997). Quality and Coherence in General Education. En: Gaff, J. G. \& Ratcliff, J. L. Handbook of the Undergraduate Curriculum (141-169). San Francisco: Jossey-Bass Publishers.

Rhoads, R.A. (1995). Critical Multiculturalism, Border Knowledge, and the Canon: Implications for General Education and the Academy. In: The Journal of General Education, 44(4), 256-273.

Tamariz, C. y Espinosa Martínez, A.C. (septiembre-octubre, 2007)). Un modelo transdisciplinario para la Universidad: marco epistémico (2a parte). Visión Docente Con-Ciencia, 7(38), 16-26.

Tubino, F. (2003). Ciudadanías complejas y diversidad cultural. En: Vigil, N. y Zariquiey, $R$. (eds.). Ciudadanías inconclusas. El ejercicio de los derechos en sociedades asimétricas. Lima, Perú: Cooperación Alemana al Desarrollo y PUCP, 167-191.

Vélez Cardona, W. (diciembre, 2011). Una educación general transdisciplinaria para el fortalecimiento de la Universidad. En: Revista Umbral, (6), 5-32.

Vélez Cardona, W. (septiembre, 2009). Gaia y la complejidad bio-social. Revista Umbral, (1), 1-12.

Walsh, C. (2007). Interculturalidad, colonialidad y educación. En: Revista Educación y Pedagogía, 9(48), 25-35.

Weislogel, E. (2011). The Transdisciplinary Imperative. Transdisciplinary Studies (1), 219-225. 
Wickson, F., Carew, A.L. y Russell, A.W. (2006). Transdisciplinarity Research: Characteristics, Quandaries and Quality. Futures, 38, 1046-1059.http://dx.doi. org/10.1016/j.futures.2006.02.011

Woidwode, C. (2013, June). New Departures in Tackling Urban Climate Change: Transdisciplinarity for Social Transformation: A Critical Appraisal of the WBGU's 2011 Report. Integral Review, 9(2), 384-412. 